\title{
Boas práticas de enfermagem a pacientes em ventilação mecânica invasiva na emergência hospitalara
}

\author{
Good nursing practices towards patients on invasive mechanical ventilation in hospital emergency \\ Buenas prácticas de enfermería a pacientes en ventilación mecánica invasiva en emergencia \\ hospitalaria
}

Cleverson dos Santos ${ }^{1}$ [C]

Eliane Regina Pereira do Nascimento ${ }^{1}$ (c) Patrícia Madalena Vieira Hermida ${ }^{1}$ (1)

Tatiana Gaffuri da Silva ${ }^{1}$ (D)

Sabrina Guterres da Silva Galetto ${ }^{1}$ (1)

Nelson Junior Cardoso da Silva ${ }^{1}$ (D)

Nádia Chiodelli Salum ${ }^{1}$ (D)

1. Universidade Federal de Santa Catarina.

Florianópolis, SC, Brasil
Autor correspondente:

Patrícia Madalena Vieira Hermida

E-mail: patymadale@yahoo.com.br

Recebido em 30/10/2019.

Aprovado em 05/12/2019.

DOI: 10.1590/2177-9465-EAN-2019-0300

\section{RESUMO}

Objetivo: Identificar os cuidados concebidos como boas práticas de enfermagem a pacientes em ventilação mecânica invasiva no contexto de emergência hospitalar. Método: Estudo descritivo, qualitativo, realizado de junho a setembro de 2017. Participaram 16 enfermeiros da emergência geral de um hospital da região Sul do Brasil. Os cuidados, extraídos da literatura e classificados quanto ao nível de evidência, foram agrupados em categorias por similaridade e selecionados nos Grupos de Discussão. Resultados: Os enfermeiros consideraram como boas práticas aos pacientes em ventilação mecânica invasiva um total de 13 cuidados, os quais estão relacionados ao tubo endotraqueal, ao ventilador e circuito, à prevenção de broncoaspiração, ao controle de infecção e à sedação, analgesia/sono, vigília/dor. Os cuidados foram extraídos de estudos com níveis de evidência Ilb, IV e VI. Conclusão e implicações para a prática: As boas práticas de enfermagem em ventilação mecânica invasiva, concebidas pelos enfermeiros da emergência, respaldam cientificamente a assistência ao paciente em suporte ventilatório invasivo, podendo ser aplicadas em contextos similares.

Palavras-Chave: Respiração Artificial; Cuidados de Enfermagem; Enfermagem em Emergência; Segurança do Paciente.

\section{ABSTRACT}

Objective: To identify care conceived as good nursing practices for patients on invasive mechanical ventilation in the context of hospital emergency. Method: Descriptive, qualitative study, conducted from June to September 2017 with the participation of 16 nurses from general emergency room of a hospital in Brazil's southern region. Care, extracted from the literature and classified according to evidence level, were grouped into categories by similarity and selected in Discussion Groups. Results: The nurses considered a total of 13 care measures as good practices for the patients on mechanical ventilation. These care measures are related to endotracheal tube, ventilator and circuit, prevention of bronchoaspiration, infection control and sedation, analgesia/sleep wakefulness/pain. Practices were extracted from studies with evidence levels Ilb, IV and VI. Conclusion and implications for practice: Good nursing practices related to invasive mechanical ventilation, as conceived by nurses from the emergency room, scientifically supportive care towards patients on invasive ventilatory support and may be applied in similar contexts.

Keywords: Respiration, Artificial; Nursing Care; Emergency Nursing; Patient Safety.

\section{RESUMEN}

Objetivo: Identificar los cuidados concebidos como buenas prácticas de enfermería a pacientes en ventilación mecánica invasiva en el contexto de emergencia hospitalaria. Método: Estudio descriptivo, cualitativo, realizado entre junio y septiembre de 2017. Participaron 16 enfermeros de la emergencia general de un hospital de la región Sur de Brasil. Los cuidados, extraídos de la literatura y clasificados según el nivel de evidencia, se agruparon en categorías por similitud y seleccionados en los grupos de discusión. Resultados: Los enfermeros consideraron como buenas prácticas para pacientes con ventilación mecánica invasiva un total de 13 cuidados, relacionados con el tubo endotraqueal, el ventilador y circuito, la prevención de la broncoaspiración, e control de infección y la sedación, la analgesia/el sueño, la vigilia/dolor. Los cuidados fueron extraídos de estudios con niveles de evidencia Ilb, IV y VI. Conclusión e implicaciones para la práctica: Las buenas prácticas de enfermería en ventilación mecánica invasiva, concebidas por enfermeros de emergencia, respaldan científicamente la atención al paciente con asistencia respiratoria invasiva y pueden aplicarse en contextos similares.

Palabras clave: Respiración Artificial; Atención de Enfermería; Enfermería de Urgencia; Seguridad del Paciente. 


\section{INTRODUÇÃO}

As Emergências Hospitalares (EH) são unidades complexas, organizadas em termos de estrutura física e de pessoal com agilidade, habilidade, destreza e capacidade de raciocinar de maneira consciente e segura no atendimento à população. ${ }^{1}$ Pacientes em estado crítico são admitidos nessas unidades e com frequência necessitam de intubação traqueal e ventilação mecânica invasiva (VMI). ${ }^{2}$ Nos Estados Unidos, anualmente centenas de milhares de pacientes são submetidos à ventilação mecânica nas EH. ${ }^{3}$ No Japão, 46,4\% dos pacientes sob esse tipo de ventilação foram exclusivamente monitorados fora do ambiente de Terapia Intensiva. ${ }^{4}$

Contudo, pneumonia associada à ventilação mecânica (PAVM) tem sido atribuída à intubação nas EH e ao tempo de permanência nesse setor. ${ }^{5}$ Como a admissão tardia na Unidade de Terapia Intensiva (UTI) de pacientes ventilados mecanicamente nas EH também está associada a maior mortalidade e utilização adicional de recursos se o paciente sobrevive, recomenda-se um tempo de espera nas EH não superior a quatro horas. ${ }^{6}$ Embora esses pacientes possam se beneficiar com a transferência imediata para uma UTI, essa opção se encontra limitada pela superlotação e recursos escassos, fato que justifica a implementação de cuidados direcionados ao paciente em ventilação mecânica em $\mathrm{EH} .{ }^{7}$

A ventilação mecânica substitui total ou parcialmente a ventilação espontânea e está indicada na insuficiência respiratória aguda (IRA) ou crônica agudizada. Quando empregada de modo invasivo é utilizado tubo endotraqueal ou cânula de traqueostomia. ${ }^{8}$ Esse tipo de suporte demanda assistência especializada da equipe multiprofissional, ${ }^{9}$ tendo o enfermeiro a responsabilidade de manter a permeabilidade das vias áreas do paciente intubado, assim como o domínio sobre os parâmetros do ventilador, necessário para avaliar a adaptação do paciente aos parâmetros e implementar os cuidados de enfermagem. ${ }^{10}$

Cuidados considerados boas práticas assistenciais no contexto das $\mathrm{EH}$ devem ser empregados, a fim de promover a segurança do paciente e contribuir para a qualidade do serviço prestado. ${ }^{11} \mathrm{Na}$ enfermagem, entende-se boas práticas como o conjunto inter-relacionado e indissociável de teorias, técnicas, processos e atividades visto como as melhores opções disponíveis para o cuidado da área, guardando consistência com conhecimentos, valores, contextos, ambientes, objetivos e evidências no interesse da saúde. ${ }^{12}$

Considerando-se a frequência de pacientes com instabilidade respiratória em uso de VMI na $\mathrm{EH}$, a possibilidade de desajustes entre o ventilador e o paciente, o risco de pneumonia associada à ventilação mecânica (PAV) e a necessidade de profissional capacitado para lidar com todas essas variações com segurança, elaborou-se este estudo, que teve como objetivo identificar os cuidados concebidos como boas práticas de enfermagem a pacientes em ventilação mecânica invasiva no contexto de emergência hospitalar.

\section{MÉTODO}

Pesquisa descritiva de abordagem qualitativa, desenvolvida na unidade de emergência de um hospital geral público da região Sul do Brasil, referência no atendimento a pacientes com trauma O estudo foi aprovado pelo Comitê de Ética em Pesquisa com Seres Humanos sob o Parecer n. 1.807.924. Os participantes assinaram o Termo de Consentimento Livre e Esclarecido.

Do total de 20 enfermeiros lotados na unidade de emergência, 16 participaram do estudo por atenderem aos critérios de inclusão: ser enfermeiro e atuar no setor há no mínimo seis meses. Foram excluídos enfermeiros atuantes em cargo administrativo, em férias ou em afastamento no período da coleta de dados. A escolha dos participantes se justifica pelo fato de o enfermeiro ser o profissional de enfermagem responsável pelo cuidado do paciente sob ventilação mecânica.

Primeiramente, um dos pesquisadores realizou um levantamento bibliográfico no período de junho a agosto de 2017, a fim de identificar estudos que abordassem cuidados ao paciente em ventilação mecânica. Para tanto, foram consultadas as Bases de Dados da Literatura Latino-Americana e do Caribe em Ciências da Saúde (LILACS), Scientific Eletronic Library Online (SciELO), Medical Literature Analysis e Retrieval System Online (MEDLINE/PubMed) e Base de Dados em Enfermagem (BDENF) com os seguintes descritores/palavras-chave: Respiração Artificial; Respiração Mecânica; Ventilação Mecânica; Suporte Ventilatório; Respirador Mecânico; Ventilador Mecânico Respiração por Aparelhos; Ventilação por Aparelhos; Cuidados de Enfermagem; Mechanical Ventilation; Artificial Respiration; Nursing Care; Respiración Artificial; Ventilación Mecánica; e Cuidados de Enfermería. Selecionou-se nove estudos para a leitura na íntegra por apresentarem cuidados de enfermagem relativos à pacientes em VMI e nível de evidência. Esses cuidados foram agrupados por similaridade em seis categorias: cuidados relacionados ao tubo endotraqueal; ao ventilador e circuito; à prevenção de broncoaspiração; ao controle de infecção; à sedação, analgesia/sono e vigília/dor.

Para eleger os cuidados considerados boas práticas para aplicação na emergência ao paciente em VMI, formou-se Grupos de Discussão em setembro de 2017, os quais ocorreram em uma sala privativa da unidade de emergência, logo após a passagem de plantão e tiveram duração média de 90 minutos. Foram organizados quatro grupos, em média com quatro enfermeiros dos períodos diurno e noturno, considerando-se a disponibilidade dos participantes. Realizou-se um encontro com cada grupo, conduzido por um dos pesquisadores. Inicialmente, foi destacado o objetivo do grupo e orientados os enfermeiros acerca da seleção dos cuidados, devendo considerar além do nível de evidência, a aplicabilidade no contexto da instituição. Em seguida, foi apresentado com recurso multimídia um quadro com os cuidados de enfermagem, o nível de evidência e as opções de resposta (Sim - possível de ser aplicado; Não - impossível de ser aplicado). Os participantes discutiram cada cuidado até obter um consenso daqueles considerados como boas práticas. 
Quadro 1. Cuidados de enfermagem por categoria e nível de evidência dos estudos, SC, Brasil, 2017.

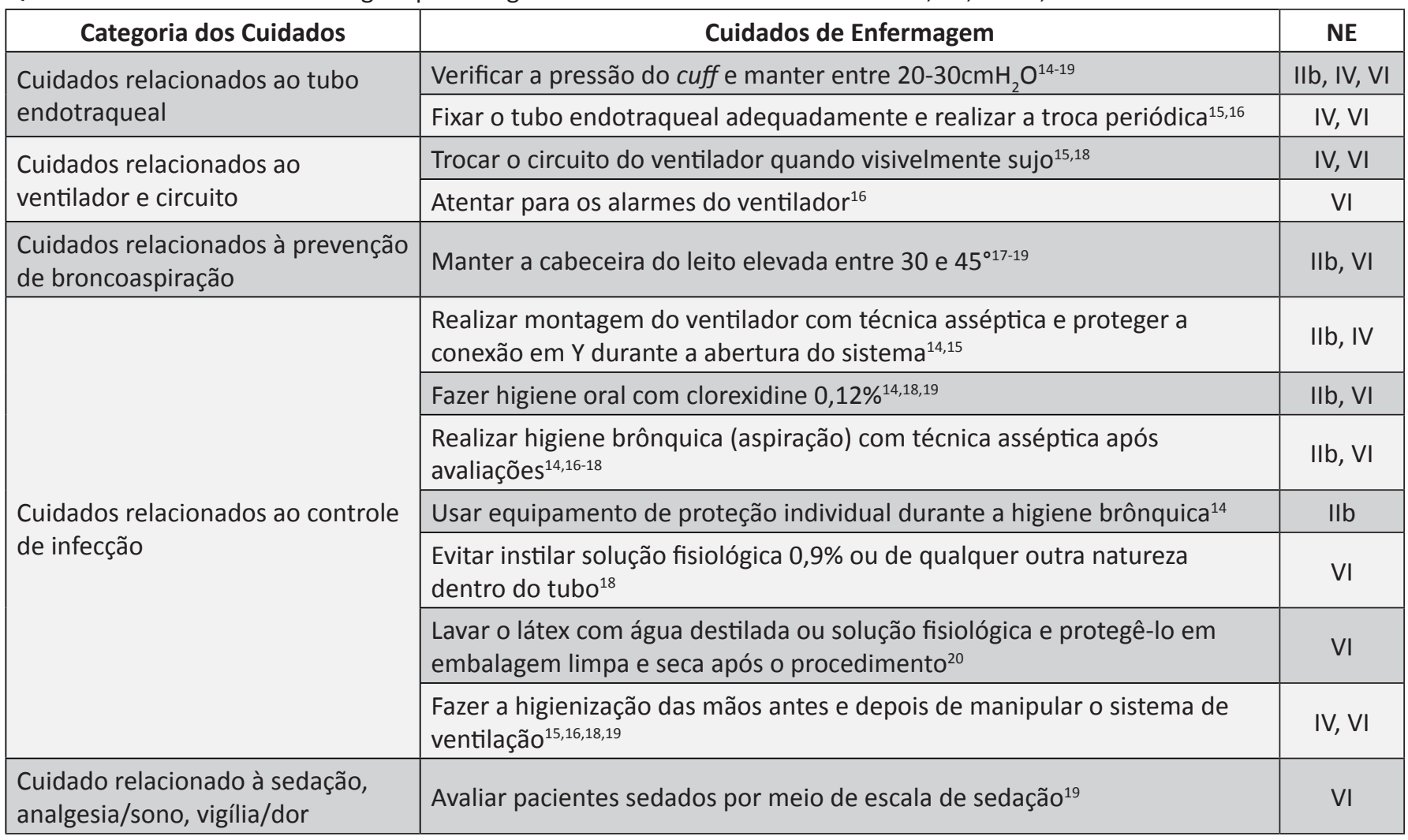

*Nota: NE: Nível de Evidência dos estudos.

Para a classificação do nível de evidência, foram considerados os tipos de estudos, representados em sete níveis: nível la: Revisão sistemática de Ensaios Clínicos Randomizados (ECR); nível lb: Revisão sistemática de ensaios não randomizados; nível Ila: ECR individual; nível Ilb: Ensaio não randomizado; nível III:Revisão sistemática de estudos de correlação/observação; nível IV: Estudo de correlação/observação; nível V: Revisão sistemática de estudos descritivos, qualitativos, fisiológicos; nível VI: Estudo descritivo/qualitativo/fisiológico/individual; e nível VII: Opinião de autoridades, comitês de especialistas. ${ }^{13}$

\section{RESULTADOS}

Do total de 16 participantes predominou o sexo feminino, o que correspondeu quatorze enfermeiros. Todos possuíam mais de seis anos de formação e dez deles tinham especialização na área de cuidados a pacientes críticos. Dos especialistas, dois tinham mestrado na área da saúde. O tempo de atuação na emergência variou de três a vinte e cinco anos.

Do total de 18 cuidados elencados, 13 foram concebidos pelos enfermeiros como boas práticas de enfermagem a pacientes em ventilação mecânica invasiva no contexto de emergência hospitalar, extraídos de estudos com níveis de evidência llb, IV e VI (Quadro 1). Foram considerados cinco cuidados não aplicáveis ao contexto do estudo: descartar condensado do circuito do ventilador quando presente; usar equipamento de proteção individual (EPI) para descartar o condensado; preferir sistema fechado ao aberto para a prevenção da PAV; utilizar tubo de aspiração subglótica para prevenir PAV; e evitar sedação desnecessária.

\section{DISCUSSÃO}

Boas práticas de enfermagem para a manutenção da oxigenação em pacientes sob ventilação mecânica invasiva na emergência foram reveladas neste estudo, a fim de promover maior segurança e prevenir eventos adversos.

Dentre os cuidados relacionados ao tubo endotraqueal, os enfermeiros destacaram a verificação e manutenção da pressão do cuff entre 20 e $30 \mathrm{cmH}_{2} \mathrm{O}$, cuidado de extrema importância na prevenção de complicações ${ }^{21}$ como lesões por compressão direta da mucosa traqueal, no deslocamento acidental do tubo e microbroncoaspiração. ${ }^{14}$ No Brasil, a Agência Nacional de Vigilância em Saúde (ANVISA) recomenda a pressão entre 25 a $30 \mathrm{cmH} 2 \mathrm{O} .^{22}$

Não há consenso na literatura sobre o nível de pressão a ser mantido no cuff, porém a média de pressão deve ser de $25 \mathrm{cmH}_{2} \mathrm{O} \cdot{ }^{14,17,23}$ Os tubos endotraqueais possuem cuff de alta pressão e baixo volume, sendo a pressão do cuff essencial no 
manejo do tubo. ${ }^{24}$ Como a mudança de posicionamento durante os cuidados de enfermagem pode modificar essa pressão, a mesma deve ser mensurada após alteração da posição do paciente e ajustada nos limites recomendados. ${ }^{21}$

Equipamentos respiratórios utilizados para o fornecimento de oxigênio e manejo das vias aéreas são responsáveis pelas mais altas taxas de lesão por pressão, relacionadas a dispositivos médicos em pacientes graves. O tubo endotraqueal é indispensável para proteger as vias aéreas em pacientes graves ventilados mecanicamente, ${ }^{24}$ mas sua fixação apertada com cadarço pode causar lesão labial e auricular. ${ }^{25}$ Estudo sobre os cuidados de enfermagem implementados para a prevenção de lesões cutâneo-mucosas associadas a dispositivos invasivos nas vias aéreas inferiores chamou atenção para o distanciamento do enfermeiro em relação ao cuidado direto mediante avaliação clínica e intervenções voltadas a esse público. ${ }^{26}$

Quanto aos cuidados direcionados ao ventilador mecânico e circuito, não se recomenda a troca rotineira do circuito, apenas quando sujo ou danificado. Para garantir o uso seguro deste dispositivo seu acondicionamento é fundamental. ${ }^{22}$ Monitorar e verificar o acionamento dos alarmes do ventilador são cuidados do enfermeiro na assistência ao paciente crítico. Um alarme é acionado sempre que os parâmetros limiares são excedidos, o que requer dos enfermeiros aderência às boas práticas de monitoração, bem como a resolução de problemas comuns em monitores, ${ }^{27}$ a fim de prevenir períodos prolongados de ventilação inadequada.

Pesquisa realizada com profissionais de enfermagem de três UTIs identificou que a maioria $(82,8 \%)$ sempre observava os alarmes do ventilador e no caso de ativação dos alarmes, $70,7 \%$ deles costumava averiguar a causa e $20,7 \%$ procurava outro profissional. ${ }^{16} \mathrm{~A}$ desatenção aos alarmes pode ter graves consequências nas condições clínicas do paciente, por isso o acionamento dos mesmos não deve ser negligenciado. Embora extraídos de estudos com níveis de evidência IV eVI, os cuidados com o ventilador e circuito foram considerados boas práticas.

O cuidado relacionado à prevenção de broncoaspiração concebido na presente investigação como boa prática de enfermagem, foi identificado em pesquisa ${ }^{17} \mathrm{com}$ nível de evidência científica llb, indicando relevância clínica. Assim, a angulação da cabeceira do leito deve ser mantida entre $30^{\circ}$ e $45^{\circ},{ }^{17}$ salvo na existência de contra-indicação. Esse cuidado, ideal para pacientes ventilados mecanicamente ${ }^{28}$ por prevenir PAV e outros eventos associados ao ventilador, é classificado como uma prática básica dada a sua simplicidade, risco mínimo, ausência de custo e potencial benefício ao paciente. ${ }^{29}$ Apesar da sua fácil aplicação, as taxas de adesão a esse cuidado têm ampla variação, sinalizando a necessidade de estratégias de sensibilização dos profissionais acerca da importância desta medida na composição dos conjuntos de boas práticas. ${ }^{30}$

Dos cuidados relacionados à prevenção de infecção, quatro se destacam pelo nível de evidencia (llb) do estudo, do qual foram extraídos: montagem do ventilador com técnica asséptica e proteção da conexão em Y durante a abertura do sistema para aspiração; higiene oral com clorexidine $0,12 \%$; higiene brônquica (aspiração) com técnica asséptica após avaliações; e uso de equipamento de proteção individual durante a higiene brônquica. ${ }^{14}$

O preparo do ventilador com técnica asséptica ${ }^{14} \mathrm{e}$ a proteção da conexão da traqueia distal na aspiração aberta são justificados pelo risco potencial de contaminações durante o procedimento. ${ }^{15}$ Assim, recomenda-se que os circuitos do ventilador e a conexão final das traqueias sejam acondicionados no próprio saco plástico que embalava o circuito. Esses cuidados são relevantes na prevenção da PAV, cujas taxas são elevadas. ${ }^{22}$

A higiene oral, uma das intervenções padrão ouro no controle da colonização da cavidade oral, inibe a formação do biofilme e com isso a invasão das vias aéreas por microrganismos causadores de infecções. ${ }^{16,22} \mathrm{Um}$ bundle de cuidados para prevenir PAV incluiu dentre os cuidados que necessitam de checagem diária dos enfermeiros, a higiene bucal com solução de clorexidine a cada 8 horas. ${ }^{31}$ Pesquisa em UTIs revelou que $84,5 \%$ dos profissionais de enfermagem realizavam higiene oral nos pacientes a cada 8, 12 ou 24 horas e que o uso da solução de clorexidine $0,12 \%$ na higienização se mostrou o método mais eficaz e que não agride a mucosa oral em pacientes ventilados mecanicamente. ${ }^{16}$ No serviço de Emergência, o conjunto de boas práticas para prevenção da PAV foi avaliado e a higiene oral apresentou conformidade inferior a $50 \%$, refletindo a insuficiência de recursos materiais e humanos, bem como a superlotação de pacientes em cuidados intensivos na Emergência. ${ }^{9}$ Esses dados sinalizam a necessidade de melhorias, considerando-se a relevância da higiene oral na prevenção de infecção, o seu baixo custo e a facilidade de realização.

A higiene da língua, muitas vezes negligenciada pelos profissionais, também deve ser realizada. Patógenos presentes no aspirado traqueal de pacientes intubados foram detectados no biofilme lingual, especialmente naqueles que apresentam PAV ou pneumonia por aspiração, sugerindo que melhorar a higiene bucal em pacientes sob VM pode reduzir as taxas de pneumonia. ${ }^{32}$ Para a remoção da placa dentária e de outros detritos dos dentes, língua e mucosa oral pode ser utilizado um swab ou escova de dentes com o mínimo de água, para reduzir o volume de qualquer material aspirado..$^{33}$

A intubação traqueal e a imobilidade imposta ao paciente pela sedação reduzem o transporte mucociliar e promovem a retenção de secreções nas vias aéreas. ${ }^{34}$ Ainda, o tubo endotraqueal facilita a colonização bacteriana da árvore traqueobrônquica e predispõe a aspiração de secreção contaminada pela diminuição do reflexo da tosse, acúmulo de secreção acima do balonete (espaço subglótico) e pela própria contaminação do tubo. ${ }^{22}$ Portanto, um importante cuidado de enfermagem ao paciente em ventilação mecânica é a aspiração de secreção endotraqueal, necessária para manter uma via aérea pérvia e facilitar a ventilação e consequente troca de gases..$^{35}$

Sobre esse cuidado de enfermagem, selecionado pelos enfermeiros como boa prática, a literatura ressalta a necessidade do enfermeiro avaliar o paciente antes da aspiração, mas também durante e após a técnica, mediante exame físico e 
monitorização, ${ }^{36}$ a fim de assegurar um procedimento seguro. Durante a aspiração devem ser avaliados: sons respiratórios, saturação de oxigênio (SpO2), coloração da pele, frequência respiratória $(\mathrm{FR})$, padrão respiratório, parâmetros ventilatórios e variáveis hemodinâmicas (caso monitoradas). ${ }^{37}$

Para a higiene brônquica pode ser utilizado o sistema fechado ou aberto de aspiração ${ }^{37}$. Recomenda-se a aspiração quando o paciente apresenta sinais clínicos de secreção traqueal como a presença de roncos ou diminuição dos sons respiratórios à ausculta pulmonar, não sendo indicada sua realização rotineira para prevenir obstrução das vias aéreas. ${ }^{38}$ Quanto à técnica propriamente dita, não deve ultrapassar 15 segundos por aspiração, ${ }^{37,38}$ a pressão de sucção não deve exceder $150 \mathrm{mmHg}$ negativos (ideal de 80 a $120 \mathrm{mmHg}$ negativos), e a hiperoxigenação com fração inspirada de oxigênio a 100\% deve ser utilizada. ${ }^{37} \mathrm{Na}$ escolha da sonda é preciso considerar que o seu diâmetro não deve ser superior a metade do diâmetro interno do tubo endotraqueal. ${ }^{37,38}$

No que se refere à utilização de equipamentos de proteção individual (EPIs) durante a técnica de aspiração, recomenda-se o uso de luvas, óculos de proteção, máscara e avental pelo profissional, ${ }^{37}$ cuidado que merece destaque pela sua relevância na prevenção de infecções relacionadas à assistência à saúde. Estudo revelou que $95 \%$ dos profissionais utilizaram EPI no momento da aspiração de secreções de pacientes sob ventilação mecânica invasiva, o que denota sensibilização para o cumprimento da técnica. Entretanto, o uso de luvas durante o procedimento teve uma menor adesão $(88,2 \%)$ dos profissionais. ${ }^{35}$ Chama-se a atenção para o uso adequado dos EPIs pelos enfermeiros, tendo em vista que este tem como atividade privativa a aspiração das vias aéreas em pacientes graves, submetidos à intubação orotraqueal ou traqueostomia, em unidades de emergência, de internação intensiva, semi-intensiva, intermediária ou demais unidades assistenciais, conforme Resolução n. 557 do Conselho Federal de Enfermagem. ${ }^{39}$

Quanto à utilização de solução fisiológica $0,9 \%$ ou de qualquer outra natureza dentro do tubo durante aspiração, deve ser evitada de maneira rotineira, ${ }^{37,40}$ tendo em vista que efeitos colaterais como diminuição da saturação de oxigênio, agitação do paciente e aumento do volume de secreções foram observados após a instilação de solução salina com aspiração. Embora esse cuidado não seja recomendado de forma rotineira pelo seu potencial prejuízo aos pacientes, ainda é realizado por enfermeiros e fisioterapeutas, de modo que a educação desses profissionais deve focar nas melhores práticas em relação à sucção do tubo endotraqueal. ${ }^{40}$

Ainda sobre os cuidados relacionados ao tubo endotraqueal, destaca-se que a lavagem do látex com água destilada ou solução fisiológica e a sua proteção ao término da aspiração com embalagem limpa e seca, são medidas de prevenção e controle de infecção do paciente em ventilação mecânica. Sobre a limpeza do sistema de aspiração e proteção do látex em embalagem limpa e seca após procedimento, estudo desenvolvido com enfermeiros revelou a adoção desta prática por mais de $92 \%$ dos profissionais da enfermagem. ${ }^{20}$
A higienização das mãos antes e depois de manipular o sistema de ventilação também foi selecionada pelos enfermeiros do presente estudo como boa prática de cuidado. Recomendada e comprovadamente impactante na prevenção de infecções respiratórias relacionadas à assistência à saúde, inclusive da PAV, a higiene das mãos esteve presente em quase metade $(47,8 \%)$ dos bundles identificados em um estudo de revisão, embora a sua adesão entre os profissionais permaneça abaixo do esperado, comprometendo a segurança do paciente. ${ }^{30}$

Para avaliar o nível de sedação daqueles que estão sob VM na Emergência Hospitalar, a proposta, extraída de estudo com nível de evidência VI, é a utilização de escalas como a de Richmond Agitation-Sedation Scale (RASS), que deve ser empregada para o ajuste da infusão medicamentosa frente à necessidade clínica do paciente, evitando o excesso de sedação e minimizando o risco de efeitos adversos induzidos pelo tratamento. ${ }^{41}$

Considera-se limitação da pesquisa a não inclusão de estudos com maior nível de evidência, uma vez que revisões sistemáticas e ensaios clínicos randomizados são padrão-ouro para a aplicação dos cuidados na prática.

\section{CONCLUSÃO E IMPLICAÇÕES PARA A PRÁTICA}

Os cuidados concebidos como boas práticas de enfermagem a pacientes em ventilação mecânica invasiva estão relacionados ao tubo endotraqueal, ao ventilador mecânico e circuito respiratório, à prevenção de broncoaspiração, ao controle de infecção e à sedação, analgesia/sono, vigília/dor. Os cuidados foram extraídos de estudos com níveis de evidência Ilb, IV e VI e respaldam cientificamente a assistência ao paciente em suporte ventilatório invasivo, podendo ser aplicados em contextos similares.

\section{CONTRIBUIÇÕES DOS AUTORES}

Concepção e desenho do estudo. Coleta, análise e interpretação dos dados. Discussão dos resultados. Redação e revisão crítica do manuscrito. Aprovação da versão final do artigo. Responsabilidade por todos os aspectos do conteúdo e a integridade do artigo publicado: Cleverson dos Santos. Eliane Regina Pereira do Nascimento. Patrícia Madalena Vieira Hermida. Análise e interpretação dos dados. Discussão dos resultados. Redação e revisão crítica do manuscrito. Aprovação da versão final do artigo. Responsabilidade por todos os aspectos do conteúdo e a integridade do artigo publicado: Tatiana Gaffuri da Silva. Interpretação dos resultados. Discussão dos resultados. Redação e revisão crítica do manuscrito. Aprovação da versão final do artigo. Responsabilidade por todos os aspectos do conteúdo e a integridade do artigo publicado: Sabrina Guterres da Silva Galetto. Nelson Junior Cardoso da Silva. Análise de dados. Redação e revisão crítica do manuscrito. Aprovação da versão final do artigo. Responsabilidade por todos os aspectos do conteúdo e a integridade do artigo publicado: Nádia Chiodelli Salun. 


\section{EDITOR ASSOCIADO}

\author{
Antonio José Almeida Filho
}

\section{REFERÊNCIAS}

1. Antonelli RC, Bellucci Jr JA. Gerenciamento de enfermagem em serviço hospitalar de emergência: revisão integrativa da literatura. Semina. 2014 jul/dez;35(2):137-46. http://dx.doi.org/10.5433/16790367.2014v35n2p137.

2. Cabrini L, Landoni G, Radaelli MB, Saleh O, Votta CD, Fominskiy E et al. Tracheal intubation in critically ill patients: a comprehensive systematic review of randomized trials. Crit Care. 2018 jan;22(6):6. http://dx.doi. org/10.1186/s13054-017-1927-3. PMid:29351759.

3. Fuller BM, Mohr NM, Roberts BW, Carpenter CR, Kollef MH, Avidan MS. Protocol for a multicentre, prospective cohort study of practice patterns and clinical outcomes associated with emergency department sedation for mechanically ventilated patients: the ED-SED Study. BMJ Open. 2018 out;8(10):e023423. http://dx.doi.org/10.1136/bmjopen-2018-023423. PMid:30344178.

4. IwashitaY, Yamashita K, Ikai H, Sanui M, Imai H, Imanaka Y. Epidemiology of mechanically ventilated patients treated in ICU and non-ICU settings in Japan: a retrospective database study. Crit Care. 2018 dez;22(1):329. http://dx.doi.org/10.1186/s13054-018-2250-3. PMid:30514327.

5. DeLuca Jr LA, Walsh P, Davidson Jr DD, Stoneking LR, Yang LM, Grall $\mathrm{KJH}$ et al. Impact and feasibility of an emergency department-based ventilator-associated pneumonia bundle for patients intubated in an academic emergency department. Am J Infect Control. 2017 fev;45(2):151 7. http://dx.doi.org/10.1016/j.ajic.2016.05.037. PMid:27665031.

6. Hung SC, Kung CT, Hung CW, Liu BM, Liu JW, Chew G et al. Determining delayed admission to intensive care unit for mechanically ventilated patients in the emergency department. Crit Care. 2014 ago;18(4):485. http://dx.doi.org/10.1186/s13054-014-0485-1. PMid:25148726.

7. Angotti L, Richards J, Fisher D, Sankoff J, Seigel T, Al Ashry H et al. Duration of mechanical ventilation in the emergency department. West J Emerg Med. 2017 ago;18(5):972-9. http://dx.doi.org/10.5811/ westjem.2017.5.34099. PMid:28874952.

8. Barbas CS, Ísola AM, Farias AM, Cavalcanti AB, Gama AM, Duarte AC et al. Brazilian recommendations of mechanical ventilation 2013. Part I. Rev Bras Ter Intensiva. 2014 abr/jun;26(2):89-121. http://dx.doi. org/10.5935/0103-507X.20140017. PMid:25028944.

9. Frota ML, Campanharo CRV, Lopes MCBT, Piacezzi LHV, Okuno MFP, Batista REA. Good practices for preventing ventilator-associated pneumonia in the emergency department. Rev Esc Enferm USP. 2019 jun;53:e0460. http://dx.doi.org/10.1590/s1980-220x2018010803460. PMid:31215615.

10. Morais Fo LA, Gue Martini J, Vargas MAO, Reibnitz KS, Bitencourt JVOV Lazzari D. Competência legal do enfermeiro na urgência/emergência. Enferm Foco. 2016;7(1):18-23. http://dx.doi.org/10.21675/2357707X.2016.v7.n1.659.

11. Paixão DPSS, Batista J, Maziero ECS, Alpendre FT, Amaya MR, Cruz EDA. Adhesion to patient safety protocols in emergency care units. Rev Bras Enferm. 2018;71(Suppl 1):577-84. http://dx.doi.org/10.1590/0034 7167-2017-0504. PMid:29562014.

12. Brandão MAG, Barros ALBL, Primo CC, Bispo GS, Lopes ROP. Nursing theories in the conceptual expansion of good practices in nursing. Rev Bras Enferm. 2019 mar/abr;72(2):577-81. http://dx.doi.org/10.1590/00347167-2018-0395. PMid:31017224.

13. Polit DF, Beck CT. Prática de enfermagem baseada em evidências: fundamentos. In: Polit DF, Beck CT, organizadores. Fundamentos de pesquisa em enfermagem: avaliação de evidências para a prática da enfermagem. 9. ed. Porto Alegre: Artmed; 2018. p. 53-82.

14. Gonçalves FAF, Brasil VV, Minamisava R, Caixeta CR, Oliveira LMAC, Cordeiro JABL. Eficácia de estratégias educativas para ações preventivas da pneumonia associada à ventilação mecânica. Esc Anna Nery. 2012 out/ dez;16(4):802-8. http://dx.doi.org/10.1590/S1414-81452012000400023.

15. Báez Figueredo RM, Samudio M. Conocimientos, actitudes y prácticas del personal de enfermería sobre medidas de prevención de neumonías asociadas a la ventilación mecánica en la unidad de cuidados intensivos de adultos del Instituto de Previsión Social. Mem Inst Investig Cienc Salud [Internet]. 2013 jun; [citado 2018 maio 19];11(1):22-30. Disponíve em: http://scielo.iics.una.py/pdf/iics/v11n1/v11n1a04.pdf

16. Melo E, Teixeira C, Oliveira R, Almeida D, Veras J, Studart R. Cuidados de enfermagem ao utente sob ventilação mecânica internado em unidade de terapia intensiva. Rev Enf Ref. 2014 fev/mar;serlV(1):55-63. http:// dx.doi.org/10.12707/RIII1316.

17. Abbasinia M, Bahrami N, Bakhtiari S, Yazdannik A, Babaii A. The effect of a designed respiratory care program on the incidence of ventilatorassociated pneumonia: a clinical trial. J Caring Sci. 2016 jun;5(2):161. PMid:27354980.

18. Silva SG, Nascimento ERP, Salles RK. Bundle to prevent ventilatorassociated pneumonia: a collective construction. Texto Contexto Enferm. 2012 out/dez;21(4):837-44. http://dx.doi.org/10.1590/S010407072012000400014.

19. Nevot MJV. Evaluación del cumplimiento de un protocolo de prevención de Neumonía asociada a Ventilación mecánica en una UCI polivalente. Enfermería Global. 2015 abr;14(2):102-17. http://dx.doi.org/10.6018/ eglobal.14.2.185231.

20. Frota OP, Loureiro MDR, Ferreira AM. Open system endotrachea suctioning: practices of intensive care nursing professionals. Esc Anna Nery. 2014;18(2):296-302. http://dx.doi.org/10.5935/1414-8145.20140043.

21. Alcan AO, Van Giersbergen MY, Dincarslan G, Hepcivici Z, Kaya E, Uyar $M$. Effect of patient position on endotracheal cuff pressure in mechanically ventilated critically ill patients. Aust Crit Care. 2017 set;30(5):267-72. http://dx.doi.org/10.1016/j.aucc.2016.11.006. PMid:27993545.

22. Agência Nacional de Vigilância Sanitária. Medidas de prevenção de infecção relacionada à assistência à saúde. Brasília: Anvisa; 2017.

23. Sole ML, Bennett M. Comparison of airway management practices between registered nurses and respiratory care actitioners. Am J Crit Care. 2014 maio;23(3):191-9, quiz 200. http://dx.doi.org/10.4037/ ajcc2014424. PMid:24786807.

24. Lizy C, Swinnen W, Labeau S, Poelaert J, Vogelaers D, Vandewoude $\mathrm{K}$ et al. Cuff pressure of endotracheal tubes after changes in body position in critically ill patients treated with mechanical ventilation. Am J Crit Care. 2014 jan;23(1):e1-8. http://dx.doi.org/10.4037/ajcc2014489. PMid:24382623.

25. Black JM, Kalowes P. Medical device-related pressure ulcers. Chronic Wound Care Manag Res. 2016;3:91-9. http://dx.doi.org/10.2147/ CWCMR.S82370

26. Pinto DM, Schons ES, Busanello J, Costa VZ. Segurança do paciente e a prevenção de lesões cutâneo-mucosas associadas aos dispositivos invasivos nas vias aéreas. Rev Esc Enferm USP. 2015 out;49(5):775-82. http://dx.doi.org/10.1590/S0080-623420150000500010. PMid:26516747.

27. Marta CB, Seabra Jr HC, Costa DJ, Martins GM, Silva RCL, Pereira LS The nursing team before alarm triggering in the neonatal intensive care unit. Care Online [Internet]. 2016 jul/set; [citado 2019 out 12];8(3):4773-9. Disponível em: http://www.seer.unirio.br/index.php/cuidadofundamental/ article/view/4054/pdf

28. Martinez BP, Marques TI, Santos DR, Silva VS, Nepomuceno Jr BR Alves GAA et al. Influence of different degrees of head elevation on respiratory mechanics in mechanically ventilated patients. Rev Bras Ter Intensiva. 2015;27(4):347-52. http://dx.doi.org/10.5935/0103507X.20150059. PMid:26761472.

29. Klompas M, Branson R, Eichenwald EC, Greene LR, Howell MD, Lee $\mathrm{G}$ et al. Strategies to prevent ventilator-associated pneumonia in acute care hospitals: 2014 update. Infect Control Hosp Epidemiol. 2014 ago;35(8):915-36. http://dx.doi.org/10.1086/677144. PMid:25026607.

30. Alecrim RX, Taminato M, Belasco AGS, Barbosa D, Kusahara DM Fram D. Good practices in the prevention of ventilator-associated pneumonia. Acta Paul Enferm. 2019 jan/fev;32(1):11-7. http://dx.doi. org/10.1590/1982-0194201900003.

31. Lim K-P, Kuo S-W, Ko W-J, Sheng W-H, Chang Y-Y, Hong M-C et al Efficacy of ventilator-associated pneumonia care bundle for prevention of ventilator-associated pneumonia in the surgical intensive care units of a medical center. J Microbiol Immunol Infect. 2015 jun;48(3):316-21. http://dx.doi.org/10.1016/j.jmii.2013.09.007. PMid:24183990. 
32. Souza LCD, Mota VBR, Carvalho AVSZ, Corrêa RGCF, Libério SA, Lopes FF. Association between pathogens from tracheal aspirate and oral biofilm of patients on mechanical ventilation. Braz Oral Res. 2017 jun;31(0):e38. http://dx.doi.org/10.1590/1807-3107bor-2017.vol31.0038. PMid:28591237.

33. Hellyer TP, Ewan V, Wilson P, Simpson AJ. The Intensive Care Society recommended bundle of interventions for the prevention of ventilatorassociated pneumonia. J Intensive Care Soc. 2016 ago;17(3):238-43. http://dx.doi.org/10.1177/1751143716644461. PMid:28979497.

34. Oliveira J, Zagalo C, Cavaco-Silva P. Prevention of ventilator-associated pneumonia. Rev Port Pneumol. 2014 maio/jun;20(3):152-61. http:// dx.doi.org/10.1016/j.rppneu.2014.01.002. PMid:24674617.

35. Cruz JRM, Martins MDS. Pneumonia associada à ventilação mecânica invasiva: cuidados de enfermagem. Rev Enf Ref. 2019 mar;serlV(20):87-96. http://dx.doi.org/10.12707/RIV18035.

36. Lopes VJ, Muller F, Souza MAR, Silva IA. Endotracheal suction in patients hospitalized in ICU with artificial airway in invasive mechanic ventilation. Rev Enferm Centro-Oeste Mineiro [Internet]. 2018 mar; [citado 2018 ago 23];8:e1973. Disponível em: http://www.seer.ufs]. edu.br/index.php/recom/article/view/1973/1905
37. Cortêz PC, Gonçalves RL, Lins DC, Sanchez FF, Barbosa No JC, Ribeiro JP. Aspiração endotraqueal de adultos intubados: evidências para boas práticas. Fisioter Bras. 2017 nov/dez;18(6):767-77. http:// dx.doi.org/10.33233/fb.v18i6.742.

38. Gonçalves RL, Tsuzuki LM, Carvalho MGS. Endotracheal suctioning in intubated newborns: an integrative literature review. Rev Bras Ter Intensiva. 2015;27(3):284-92. http://dx.doi.org/10.5935/0103-507X.20150048. PMid:26465249.

39. Resolução COFEN nº 557/2017 (BR). Normatiza a atuação da equipe de enfermagem no procedimento de aspiração de vias aéreas. Diário Oficial da União, Brasília (DF), 5 set 2017.

40. Leddy R, Wilkinson JM. Endotracheal suctioning practices of nurses and respiratory therapists: how well do they align with clinical practice guidelines? Can J Respir Ther. 2015;51(3):60-4. PMid:26283870.

41. Namigar T, Serap K, Esra AT, Özgül O, Can ÖA, Aysel A et al The correlation among the Ramsay sedation scale, Richmond agitation sedation scale and Riker sedation agitation scale during midazolam-remifentanil sedation. Rev Bras Anestesiol. 2017 jul/ ago;67(4):347-54. http://dx.doi.org/10.1016/j.bjan.2017.03.006. PMid:28412050.

\footnotetext{
a Este estudo foi extraído da dissertação de mestrado intitulada "Procedimento Operacional Padrão para cuidados de enfermagem à paciente sob ventilação mecânica invasiva na emergência hospitalar", de autoria de Cleverson dos Santos, em 2018, no Programa de Pós-Graduação Gestão do Cuidado - modalidade Profissional, da Universidade Federal de Santa Catarina. http://tede.ufsc.br/teses/PGCF0098-D.pdf
} 